\title{
9 Expectations and the Multiplier-Accelerator Model
}

\author{
Marji Lines and Frank Westerhoff
}

\subsection{Introduction}

In this paper we investigate how a simple expectations mechanism modifies the basic dynamical structure of the multiplier-accelerator model due to Samuelson (1939). Consumption depends on the expected value of present income rather than lagged income. National income is determined as a nonlinear mix of extrapolative and reverting expectations formation rules (prototypical predictors used in recent literature on financial markets). The total level of economic activity depends endogenously on the proportion of agents using the predictors.

The very simplicity of Samuelson's descriptive macroeconomic model makes it an excellent candidate for studying the effects of introducing expectations without changing the emphasis of the formalization. That is, agents' expectations are not part of an optimization problem and the resulting framework remains in the class of descriptive models. (For bibliographical references of past and recent extensions to Samuelson's model see Westerhoff (2005) and the bibliographies in other chapters of this volume.)

The expectations hypotheses follow in the style of Kaldor. Some destabilizing force exists for values near the equilibrium but the economy neither explodes nor contracts indefinitely due to a global stabilizing mechanism that is activated when the economy deviates too much from its equilibrium. These interacting forces permit a greater variety of attracting sets including point equilibria above and below the (unique) Samuelsonian equilibrium and closed curves on which lie both quasiperiodic and periodic cycles. Moreover, under realistic values for the multiplier and coefficient of acceleration, a larger area of the parameter space is characterized by stable limit sets and much of that is dominated by solutions with persistent fluctuations. 
The remainder of the paper is organized as follows. Section 2 reconsiders Samuelson's business cycle model. In section 3, we discuss the hypotheses introduced to describe expectations formation and aggregation rules. In section 4 we study the properties of the model using the local linear approximation. In section 5 we use analysis and numerical simulations to study the global properties of the model. In section 6 conclusions are offered.

\subsection{The multiplier and the accelerator}

Samuelson's seminal model incorporates the Keynesian multiplier, a multiplicative factor that relates expenditures to national income and the accelerator principle whereby induced investment is proportional to increases in consumption. An increase in investment therefore leads to an increase in national income and consumption (via the multiplier effect) which in turn raises investment (via the accelerator process). This feedback mechanism repeats itself and may generate an oscillatory behavior of output. It may also lead to explosive oscillation, monotonic convergence to an equilibrium point or monotonic divergence, depending on the values of the marginal propsensity to consume and the acceleration coefficient (See Gandolfo 1996 for a complete treatment of the dynamics over parameter space).

The assumptions are well-known. Consumption in period $t$ depends on national income in period $t-1$

$$
C_{t}=b Y_{t-1} \quad 0<b<1
$$

where $b$ is the propensity to consume out of previous period income. Invest-

ment is partly autonomous and independent of the business cycle, denoted $I_{a}$, and partly induced, proportional to changes in consumption with acceleration coefficient, $k$ :

$$
I_{t}=I^{a}+k\left(C_{t}-C_{t-1}\right) \quad k>0 .
$$

The equilibrium condition for a closed economy is

$$
Y_{t}=C_{t}+I_{t}
$$

Combining (1), (2) and (3), we obtain a second-order linear difference equation, in the income variable:

$$
Y_{t}=I_{a}+b(1+k) Y_{t-1}-b k Y_{t-2}
$$


That is, current national income depends on autonomous investment and on the output of the previous two periods. The fixed point of (4), the long-run equilibrium output, is determined as

$$
\bar{Y}=\frac{1}{1-b} I_{a}
$$

with $1 /(1-b)$ the multiplier. It follows from (1) and (2) that the other equilibrium values are $\bar{C}=b \bar{Y}$ and $\bar{I}=I_{a}$. It can be shown that stability of the fixed point requires

$$
b<\frac{1}{k}
$$

It can also be shown that no improper oscillations occur and that the flutter boundary, between monotonic and oscillatory solutions, is $b=4 k /(1+$ $k)^{2}$. With only two parameters the dynamics over parameter space are easily determined. Damped oscillations occur only in the area with $b<1 / k$ and $b<4 k /(1+k)^{2}$. In that case temporary business cycles arise due to the interplay of the multiplier and the accelerator, increased investment increases output which, in turn, induces increased investment.

A major criticism of linear business cycle theory is that changes in economic activity either die out or explode (persistent cycles only occur for a nongeneric boundary case). In reaction to this deficiency the nonlinear theory of business cycle has developed. In particular, in the seminal work of Hicks (1950) the evolution of an otherwise explosive output path was limited by proposing upper and lower bounds for investment, so-called ceilings and floors. These simple frameworks of Samuelson and Hicks are still used as workhorses to study new additional elements that may stimulate business cycles (see, besides the current monograph, Hommes 1995 and Puu, et al. 2004).

\subsection{Expectations}

As argued by Simon (1955), economic agents are boundedly rational in the sense that they lack knowledge and computational power to derive fully optimal actions. Instead, they tend to use simple heuristics which have proven to be useful in the past (Kahneman, Slovic and Tversky 1986). Survey studies reveal that agents typically use a mix of extrapolative and reverting expectation formation rules to forecast economic variables (Ito 1990, Takagi 1991). Similar results are observed in asset pricing experiments. For instance, Smith (1991) and Sonnemans et al. (2004) report that financial market participants 
typically extrapolate past price trends or expect a reversion of the price towards its long-run equilibrium value. Indeed, the dynamics of group expectations have successfully been modeled for financial markets. Contributions by Day and Huang (1990), Kirman (1993), de Grauwe et al. (1993), Brock and Hommes (1998) or Lux and Marchesi (2000) demonstrate that interactions between heterogeneous agents who rely on heuristic forecasting rules may cause complex financial market dynamics, as observed in actual markets.

Our goal is to investigate the importance of expectations for the variability of output. Our main modification of Samuleson's model is that the agents' consumption depends on their expected current income (and not on their past realized income). Note that Flieth and Foster (2002) and Hohnisch et al. (2005) model socioeconomic interactions between heterogeneous agents to explain the evolution of business confidence indicators. Both papers are able to replicate typical patterns in the German business-climate index (the so-called Ifo index), yet refrain from establishing a link between expectations and economic activity. We believe, however, that mass psychology, expressed via expectations and visible in business confidence indicators, is a major factor that may cause swings in national income. For example, new era thinking may lead to optimistic self-fulfilling prophecies (e.g. the New Economy hype) while general pessimism may cause economic slumps (Shiller 2000).

Then, with respect to Samuelson's hypothesis that consumption depends on last period's income (1), we assume that consumption depends on the expected value of current income, which is based on information available last period:

$$
C_{t}=b E_{t-1}\left[Y_{t}\right]
$$

The aggregate expectation $E_{t-1}\left[Y_{t}\right]$ is formed as a weighted average of extrapolative (denoted 1) and reverting (denoted 2) expectations:

$$
E_{t-1}\left[Y_{t}\right]=w_{t} E_{t-1}^{1}\left[Y_{t}\right]+\left(1-w_{t}\right) E_{t-1}^{2}\left[Y_{t}\right] \quad 0<w<1 .
$$

Expectations are formed with reference to a "long-run" equilibrium which is taken to be the fixed point of Samuelson's linear model, denoted in what follows as $\mathcal{Y}=I_{a} /(1-b)$. In the extrapolative expectation, or trend, formation rule, agents either optimistically believe in a boom or pessimistically expect a downturn. Such expectations are formalized as

$$
E_{t-1}^{1}\left[Y_{t}\right]=Y_{t-1}+\mu_{1}\left(Y_{t-1}-\mathcal{Y}\right) \quad \mu_{1}>0 .
$$


If output is above (below) its long-run equilibrium value, $\mathcal{Y}$, people think that the economy is in a prosperous (depressed) state and thus predict that national income will remain high (low) (a similar assumption has been applied by Day and Huang 1990).

Equilibrium-reverting expectations are formed as

$$
E_{t-1}^{2}\left[Y_{t}\right]=Y_{t-1}+\mu_{2}\left(\mathcal{Y}-Y_{t-1}\right) \quad 0<\mu_{2}<1
$$

where $\mu_{2}$ captures the agents' expected adjustment speed of the output towards its long-run equilibrium value.

The more the economy deviates from $\mathcal{Y}$, the less weight the agents put on extrapolative expectations. Agents believe that extreme economic conditions are not sustainable. Formally, the relative impact of the extrapolative rule depends on the deviation of income from equilibrium at the time that expectations are formed:

$$
w_{t}=\frac{1}{1+\left(\gamma\left(\frac{Y_{t-1}-\mathcal{Y}}{\mathcal{Y}}\right)\right)^{2}} \quad \gamma>0
$$

with $\gamma$ as a scale factor. The percentage gap is typically less than one which, when squared, results in a small number. Setting $\gamma>1$ increases the weight factor, resulting in a more realistic distribution between extrapolative and equilibrium-reverting expectations. (For example, if $\gamma=10$ and the percentage gap is $10 \%$, the proportion of agents using $E^{1}$ is $50 \%$; the proportion is $99 \%$ for $\gamma=1$.) Extrapolative and reverting expectations are linear functions of the previous level of national income, but the expectation operator, combining the heterogeneous expectations through a nonlinear weighting function, is not. In Figure $1 w_{t}$ and $1-w_{t}$, the weights given to each type of expectation are plotted against national income $(\gamma=10, b=0.8$, $\mathcal{Y}=5000$ ). Close to equilibrium the trend-following expectation dominates (and at $Y_{t}=\mathcal{Y}, w_{t}=1$ ), acting as a destabilizing force for any small deviation from the long-run equilibrium. Expectations are equally distributed (with $\gamma=10$ ) at a $10 \%$ gap between actual and long-run values of income. At further distances from $\mathcal{Y}$ the reverting expectation dominates, acting as a global stabilizing force.

Other weighting functions and other basic types of expectation formation rules can be found in, e.g., Brock and Hommes (1997, 1998). The former paper explores the expectation formation of heterogeneous producers in cobweb markets while the latter paper investigates the selection of forecasting rules among financial market participants. However, the essential 
idea is the same. For similar states of the current economy (market) agents have differing expectations about the future state, these expectations feedback through the economy (market), but the aggregate expected value is not necessarily equal to the (deterministic) value of that future state. It is also typically assumed that extremes will be considered unsustainable, providing a global mechanism for stability. This new approach to modeling how agents incorporate future uncertainty in their decision-making process breaks with both the rational expectations hypothesis and with earlier homogeneous, aggregate expectation hypotheses that R.E. criticized. Of course, assumptions about agent's expectations must be coherent with the particular context, but we argue that for business cycle theory our approach may provide a reasonable alternative.

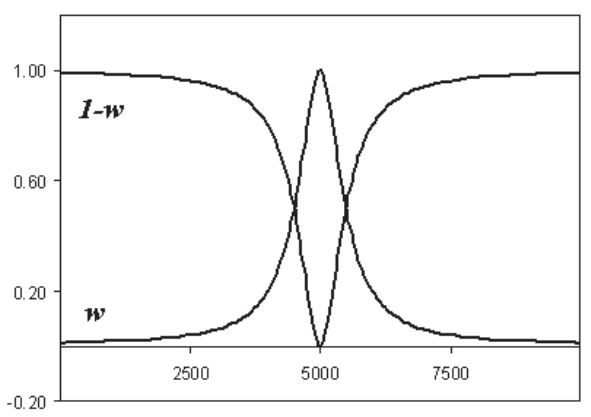

Figure 1: Weights against national income: $w$ extrapolative, $1-w$ reverting.

Substituting (2) and (7) into (3) we derive the expectations version of (4) as

$$
Y_{t}=I_{a}+b(1+k) E_{t-1}\left[Y_{t}\right]-b k E_{t-2}\left[Y_{t-1}\right]
$$

Then using (8)-(11) we arrive at a second-order nonlinear difference equation $Y_{t}=f\left(Y_{t-1}, Y_{t-2}\right)$. For the analysis we introduce an auxiliary variable $Z_{t}=Y_{t-1}$, deriving a first-order system in $\left(Y_{t}, Z_{t}\right)$ (see the Appendix for full system and Jacobian)

$$
\begin{aligned}
Y_{t} & =I_{a}+b(1+k) E_{t-1}\left[Y_{t}\right]-b k E_{t-2}\left[Z_{t-1}\right] \\
Z_{t} & =Y_{t-1}
\end{aligned}
$$


with Jacobian matrix

$$
J(Y, Z)=\left(\begin{array}{cc}
b(1+k) \frac{d E_{t-1}\left[Y_{]}\right.}{d Y_{t-1}} & -b k \frac{d E_{t-2}\left[Z_{t}\right]}{d Z_{t-1}} \\
1 & 0
\end{array}\right)
$$

\subsection{Local dynamics}

In this section we consider fixed points and the conditions for which local stability is lost. It can be shown that the equilibrium value for Samuelson's multiplier-accelerator model is also an equilibrium for the modified model. At $\mathcal{Y}$ the trend followers are predicting perfectly, $w_{1}=1$ and the Jacobian, calculated at that value, simplifies to:

$$
J(\mathcal{Y})=\left(\begin{array}{cc}
b(1+k)\left(1+\mu_{1}\right) & -b k\left(1+\mu_{1}\right) \\
1 & 0
\end{array}\right)
$$

with trace $\operatorname{tr} J=b(1+k)\left(1+\mu_{1}\right)$ and determinant $\operatorname{det} J=b k\left(1+\mu_{1}\right)$. We can use the stability conditions for a two-dimensional system to help understand how the equilibrium might lose its local stability:

$$
\begin{aligned}
& 1+\operatorname{tr} J(\mathcal{Y})+\operatorname{det} J(\mathcal{Y})>0 \quad(i) \\
& 1-\operatorname{tr} J(\mathcal{Y})+\operatorname{det} J(\mathcal{Y})>0 \quad(i i) \\
& 1-\operatorname{det} J(\mathcal{Y})>0 . \quad(i i i)
\end{aligned}
$$

The first condition holds always and we should not expect to see flip bifurcations. The second condition and third conditions, which reduce to, respectively:

$$
b<\frac{1}{1+\mu_{1}} \quad \text { and } \quad b<\frac{1}{k\left(1+\mu_{1}\right)}
$$

are not necessarily satisfied, leaving open the possibility of both fold and Neimark-Sacker bifurcations. The parameter assumptions are simply that $\mu_{1}, k>0$ and the binding inequality is condition (ii) if $k<1$, condition (iii) if $k>1$.

In Samuelson's linear model the stability conditions are satisfied always, except for the third which requires $b<1 / k$. In the linear case, of course, there is only one equilibrium set and it is a fixed point, so that when stability is lost the system itself is unstable. In the nonlinear case a fixed point may lose stability at the parameter value for which some other limit set becomes 
an attractor or there may be co-existing attractors which are limit sets for different collections of initial conditions. In the case of the Neimark-Sacker bifurcation, when the third condition is broken, global stability may continue in the form of an attractor which is a sequence of points lying on a closed curve. If attracting (and we see below that they are), these sequences represent endogenous fluctuations which are a generic feature of the dynamics (rather than the particular case of constant amplitude oscillations in Samuelson's model).

If the accelerator coefficient is less than unity, the breaking of the second condition leads to a pitchfork bifurcation, that is, as $\mathcal{Y}$ loses stability 2 new (stable) fixed points appear. These are determined by returning to the second order difference equation (12) which, setting $Y_{t-1}=Z_{t-1}=\bar{Y}$ becomes

$$
\bar{Y}=\mathcal{Y}+b(\bar{Y}-\mathcal{Y})\left(\bar{w}\left(\mu_{1}+\mu_{2}\right)+1-\mu_{2}\right)
$$

with equilibrium weight

$$
\bar{w}=\frac{\mathcal{Y}^{2}}{\mathcal{Y}^{2}+\gamma^{2}(\bar{Y}-\mathcal{Y})^{2}} .
$$

Expanding and simplifying (16) gives

$$
(\bar{Y}-\mathcal{Y})^{2}=\frac{\mathcal{Y}^{2}\left(b\left(1+\mu_{1}\right)-1\right)}{\gamma^{2}\left(b\left(\mu_{2}-1\right)+1\right)} .
$$

These two fixed points are complex-valued for $b<1 /\left(1+\mu_{1}\right)$ and become real and equal in value to $\mathcal{Y}$ at the critical value $b=1 /\left(1+\mu_{1}\right)$. For $b>1 /\left(1+\mu_{1}\right)$ there are two positive, real equilibria determined by (17), one larger and one smaller than $\mathcal{Y}$, respectively $\bar{Y}_{1}, \bar{Y}_{2}$, each attracting over a given basin, a situation of bi-stability. With these basics in mind we now turn to a study of the global dynamics using a combination of analysis and numerical simulations.

\subsection{Global dynamics}

Consider first a comparison of the dynamics over the parameter space $(k, b)$. In Figure 2, left, Samuelson's linear model is characterized by a single fixed point, stable to the left of the stability frontier $b=1 / k$, unstable to the right. At the boundary crossing the fixed point is a focus, adjacent to the left are damped oscillations (in gray), adjacent to the right explosive fluctuations (in black). The existence of any kind of persistent fluctations is guaranteed only 
for those combinations of parameter values that are on the stability frontier itself, that is for $b k=1$.

In Figure 2, right, we have the same parameter space for the expectations version of the multiplier-accelerator with standard constellation $\mu_{1}=$ $\mu_{2}=0.5, I_{a}=1000, \gamma=10,\left(Y_{0}, Z_{0}\right)=(4000,4000)$ and infinity set at $10^{10}$, transients at 5000 with maximal period 24 and precision epsilon set at 1 . This and all following plots were produced with the open-source software iDMC - Copyright Marji Lines and Alfredo Medio, available at www.dss.uniud.it/nonlinear.

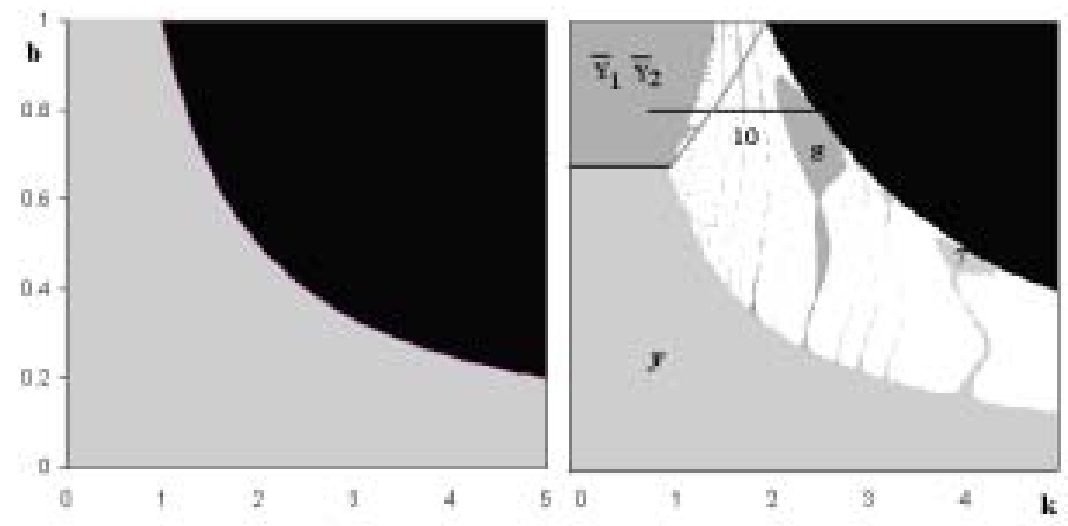

Figure 2: Parameter space $(k, b)$ : left, linear model; right, with expectations.

The black area again represents the lack of any attracting finite limit set, and the gray area on the left again represents stable fixed points. The lighter area in the middle section is characterized by quasi-periodic or high-order periodic fluctuations, in white, and cycles of the given periods in grays. For both the original and the expectation models higher values of the multiplier and the accelerator lead to instability. An economy with high demand resulting from spending most of its income on consumption encourages entrepreneurs to invest in order to keep up the supply of these goods and services. As a consequence the economy heats up. The acceleration coefficient is a reaction parameter, how strongly investment responds to changes in demand. It can also be interpreted as the capital-output ratio, how much new capital will be necessary to produce the increased output. When Samuelson was modeling the interaction between the multiplier analysis and the principle of acceleration in the late thirties the propensity to consume was 
much lower (and not only due to the Great Depression but also to spending habits), as was the captial-output ratio. Consumption out of income in the United States today has almost reached the upper bound of $b=1$, creating growth not only in the US but in the economies that supply it with goods and services such as China and India. Of course there are other issues involved, but if these interactions are fundamental and their dynamics are well-approximated by the models, the sustainability of the current situation in the U.S. is doubtful.

A noticeable difference in the model dynamics is that the area of attractors is much larger for the expectations version and that there is a significant area of attractors characterized by fluctuations (a pertinent issue for business cycle models). On the other hand, the area for which $\mathcal{Y}$ is stable (below the second condition, the line $b=0.6 \overline{6}$, and to the left of the third condition, $\left.b=0.6 \overline{6} / k_{n s}\right)$ is smaller than that of Samuelson's model. In both models there is some trade-off between the accelerator coefficient and the propensity to consume out of income for maintaining stability, and high values are de-stabilizing for both. The extreme simplicity of the dynamics in the linear version ( $\mathcal{Y}$ is stable or unstable) is replaced by more challenging dynamics, but $\mathcal{Y}$ (through its stable and unstable manifolds) remains crucial to their explanation.

For $b<0.6 \overline{6}$ stability of $\mathcal{Y}$ is lost through a Neimark-Sacker bifurcation. Fixing $b$ a constant and increasing $k$ so as to cross through the curve of the stability frontier at $b_{n s}=0.6 \overline{6} / k$, we have $\mathcal{Y}$ changing from a stable focus to an unstable focus as, simultaneously, an invariant closed curve is created (denoted, generically, as $\Gamma$ ). As $k$ is further increased the periodic or quasiperiodic limit sets on $\Gamma$ continue to be attracting over a large interval until the stability frontier for $\Gamma$ is reached, after which no attractors exist.

For $b \in(0.6 \overline{6}, 1)$, stability of $\mathcal{Y}$ is lost through a pitchfork bifurcation at the critical value $b_{p}=0.6 \overline{6}$ which has been traced in Figure 2, right to separate the subspace characterized by stable fixed point $\mathcal{Y}$ from that characterized by stable fixed points $\bar{Y}_{1}, \bar{Y}_{2}$. The bifurcation scenario moving right from the upper sub-space is more relevant for economics as a typical range for the propensity to consume out of expected income is $b \in(0.75,1)$. For small $k$ there are the two co-existing fixed points which are attractors, each with its own basin of attraction, $B\left(\bar{Y}_{1}\right), B\left(\bar{Y}_{2}\right)$ (that is, initial conditions determine on which point the trajectory comes to rest). These lose stability as $k$ is increased and a region of periodic or quasiperiodic attractors gives way to no attractors at all for larger values of the acceleration coefficient. Using the standard constellation the $(k, b)$ combination at which $\bar{Y}_{1}$ and 
$\bar{Y}_{2}$ lose stability due to a Neimark- Sacker bifurcation can be calculated as $k\left(1.5 b-3+\frac{2}{b}\right)=1$. These critical values are represented in Figure 2, right, by the curve extending from $(1,0.66 \overline{7})$ to $(2,1)$.

Let $b=0.8$. Given the standard parameter values, local properties of the fixed points can be calculated. First, $\mathcal{Y}$ is a saddle point and remains so for at least up to $k=5$, let $\lambda_{1}>1$ and $\lambda_{2}<1$. The two equilibria of the pitchfork bifurcation also exist and we have, increasing from $k=0: \bar{Y}_{1}, \bar{Y}_{2}$ are stable nodes, then (near $k=0.3$ ) they become stable foci. These fixed points lose stability through a Neimark-Sacker bifurcation at $k=1 / b \Phi \approx 1.43$.
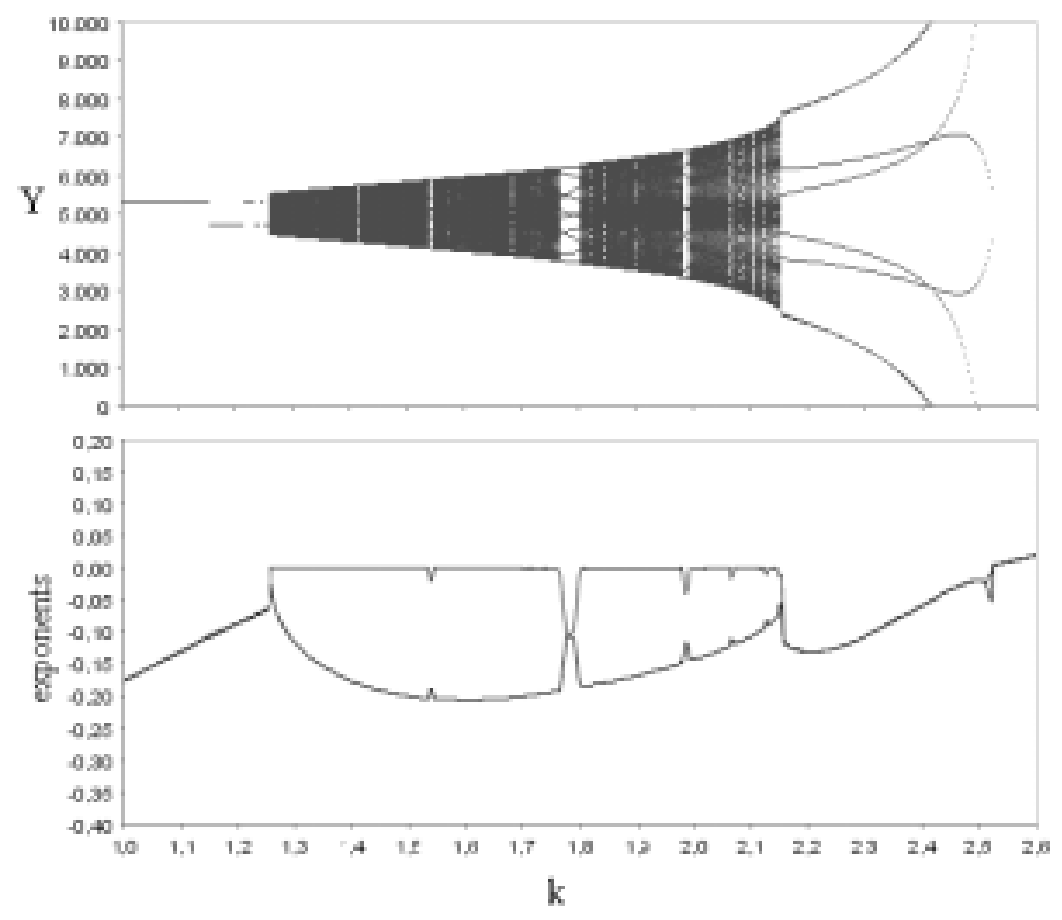

Figure 3: Above, bifurcation diagram; below, Lyapunov exponents.

Numerical simulations of the dynamics of the economy, with these parameter values, are provided in Figure 3; the single parameter bifurcation diagram for $k \in(1,2.6)$, above; the Lyapunov exponents over the same interval, below. Both figures suggest that there are three basic types of longrun dynamics and that for trajectories beginning at $(4000,4000)$ the changes occur at around $k=1.26$ and $k=2.13$. For small values of the acceleration 
coefficient the economy experiences bi-stability. The weight in the economywide expectation operator is not a function of $k$ and, for the given parameter values, $75 \%$ expect the trend to continue while $25 \%$ expect reversion. The economy moves toward one of the two fixed points, far from the Samuelsonian equilibria, and switching between high and low equilibrium values increases with $k$. Over the next interval, approximately $k \in(1.26,2.13)$ the economy is characterized by persistent fluctuations over a range of values symmetrical around $\mathcal{Y}$. For some values the recurrent behavior seems cyclical (cycles of 10 are evident), but for most the motion is quasiperiodic or periodic of order greater than 24. The last type of behavior is found in the tentacles of the octopus, period- 8 cycles that cover a wider span of national income than the invariant cycle that preceded it. The periodic cycle loses stability at around $k=2.55$ after which no attractor exists.

There are 3 puzzles to explain in this bifurcation scenario: the increased switching between $\bar{Y}_{1}$ and $\bar{Y}_{2}$; the attracting curve appearing before the critical value; the period-8 cycle which does not seem to derive from frequencylocking.

The switching behavior of the economy occurs because of the pitchfork bifurcation and bi-stability that exists for $k$ small. The switching between long-run behavior increases because as $k$ changes the separtrix, the boundary separating basins of attraction, becomes increasingly entwined. This phenomena can be seen in Figure 4 which presents the basins of attraction for the fixed points in the state space $Y \in(4000,6000)$ under the standard constellation.

Moving clockwise from upper-left $k$ increases through $0.2\left(\bar{Y}_{1}, \bar{Y}_{2}\right.$ stable nodes), $0.9,1.1,1.255\left(\bar{Y}_{1}, \bar{Y}_{2}\right.$ stable foci). Recall that initial conditions used in Figure 2 are $(4000,4000)$, the lower-left hand corner of the basin plots. The other dynamical puzzles are not so clear. In fact, on the basis of local evidence and the single and double parameter bifurcation diagrams alone, we cannot explain the large curve $\Gamma$ appearing at a value of $k$ less than the critical value of the Neimark-Sacker bifurcation of $\bar{Y}_{1}, \bar{Y}_{2}$ and the origin of the period-8 cycle, lying as it does outside the bounds of the invariant circle. The global bifurcation scenarios that answer these questions are described by Agliari, Bischi and Gardini in Chapter 1, to which we refer the reader (see, also, the business cycle application by Agliari and Dieci in Chapter 8). We consider each of these puzzles in turn.

From foci to invariant curve. An important point to note is that, although over the interval of interest the Samuelsonian fixed point has already lost local stability through a pitchfork bifurcation, the saddle point $\mathcal{Y}$ is still a 
significant factor in the global dynamics through its stable and unstable manifolds. In fact, it is the stable manifold $w^{s}(\mathcal{Y})$ (associated with $\lambda_{2}$ ) that plays the role of separatrix for the basins of attraction of the stable foci $\bar{Y}_{1}, \bar{Y}_{2}$. The unstable manifold $w^{u}(\mathcal{Y})$ (associated with $\lambda_{1}$ ) has two branches, each exiting $\mathcal{Y}$ and connecting to either $\bar{Y}_{1}$ or $\bar{Y}_{2}$ until the basins become disjoint.
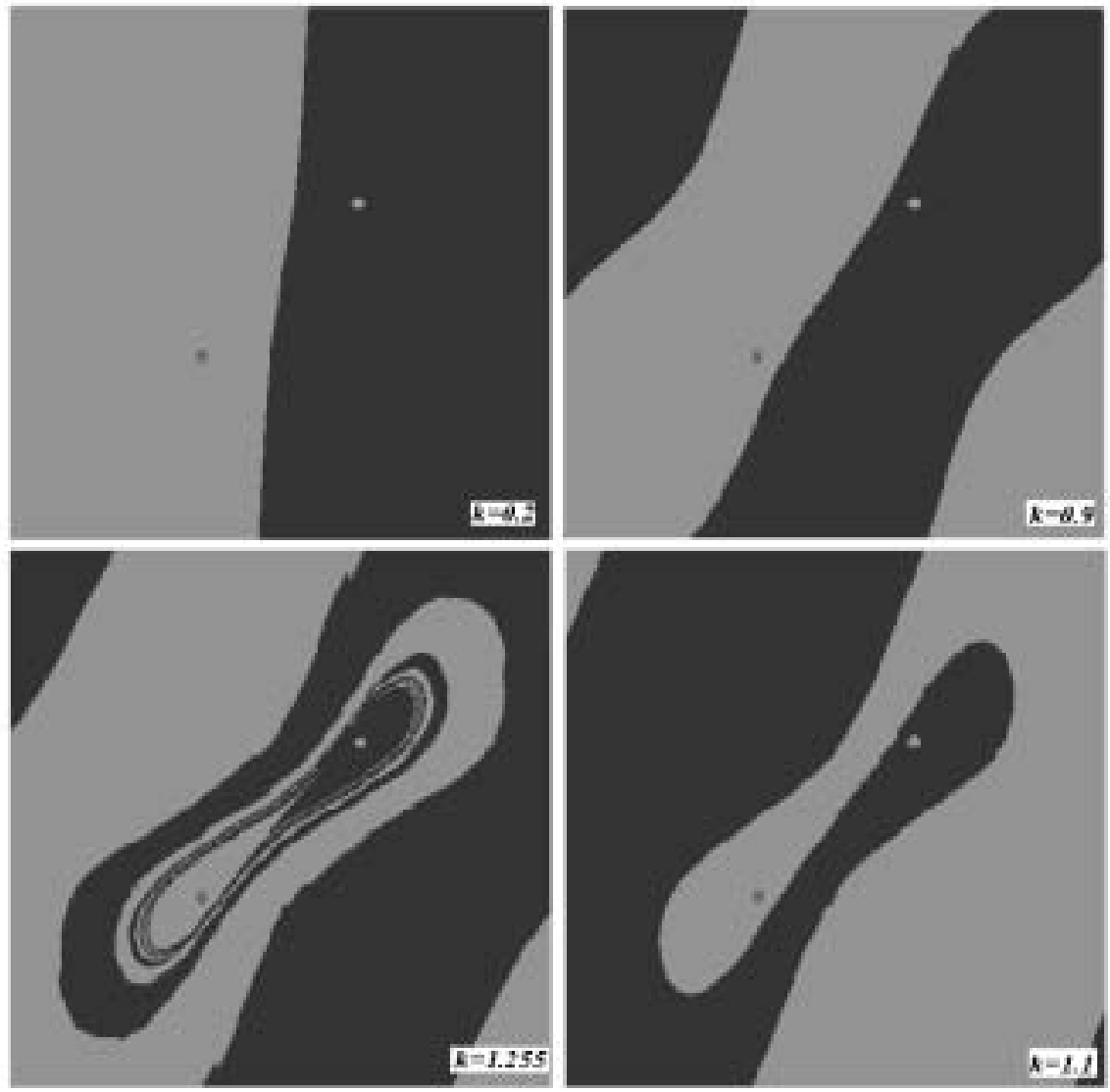

Figure 4: Basins of attraction in state space as $k$ increases.

Another point is that when there are co-existing attractors and global changes in the dynamics, bifurcation diagrams calculated on the basis of a single initial condition cannot tell the whole story. In Figure 5 we use a series of simulations of the state space to help describe what is happening over the interval $k \in(1.25,1.43)$, moving clockwise as $k$ increases, $k=$ 
1.25, 1.27, 1.35, 1.42. Again both axes are $Y \in(4000,6000)$, symmetric around $\mathcal{Y}=5000$, and the initial conditions used in Figure 3 simulations are in the lower axes' intersection. In the upper-left figure the separatrix $w^{s}(\mathcal{Y})$ separates the state space into basins of attraction for $\bar{Y}_{1}, \bar{Y}_{2}$. The convolutions of the stable manifold form a ring of entwined basins around the fixed points where, increasing $k$, an attracting invariant closed curve appears. At the creation of the attracting curve, call it $\Gamma_{s}$, a second curve, $\Gamma_{u}$, also appears which is enclosed in the first and repelling. The latter forms the separatrix between collections of initial conditions with trajectories tending to one or other of the stable foci and initial conditions with trajectories tending to the attracting $\Gamma$. As $k$ is further increased the radius of $\Gamma_{s}$ increases while that of $\Gamma_{u}$ decreases and the basins of $\bar{Y}_{1}, \bar{Y}_{2}$ contract. Between upper and lower right the basins become disjoint through a homoclinic bifurcation. Finally, the subcritical Neimark-Sacker bifurcation for $\bar{Y}_{1}, \bar{Y}_{2}$ occurs for a value of $k$ just beyond that in Figure 5, lower left, and the basins disappear altogether.

There are a number of global bifurcations involved in this interval. First, and most mysterious, is the creation of the attractor $\Gamma_{s}$, which comes to co-exist with the stable foci, and the separatrix $\Gamma_{u}$ defining its basin of attraction. The likely sequence leading to the formation of $\Gamma_{s}$ is that proposed in Chapter 1, Section 7 which we summarize as follows. In the vicinity of the tightly woven basins, where the stable manifold is coiled like yarn on a spindle, at a certain parameter value (in this case around $k=1.259$ ) a saddle-node bifurcation leads to a saddle cycle of high period along with a node cycle of the same period. The periodic points of the node immediately become repelling foci. In quick succession, over a narrow interval of $k$, we have the following changes. The periodic points are joined through a saddle connection of the outwards branches of stable manifolds of point $i$ and unstable manifolds of point $j$ forming an unstable saddle-focus connection $\Gamma_{u}$ surrounded by an attracting invariant curve $\Gamma_{s} . \Gamma_{u}$ is destroyed as a second heteroclinic loop forms from the connection of the inward stable branches of point $j$ and the inward unstable branches of point $i$ and this unstable saddlefocus connection becomes $\Gamma_{u}$, the separatrix in Figure 5.

All initial conditions outside of $\Gamma_{u}$ are attracted to the invariant curve and any economy beginning from these values (or after being disturbed to them) is destined to a recurrent fluctuation, even though there are three equilibria within the closed curve, two of which are stable. Only trajectories with initial conditions on the inside of $\Gamma_{u}$, a small area of the state space, tend to $\bar{Y}_{1}$ or $\bar{Y}_{2}$ with damped oscillations. Looking back at Figure 3 it can be observed that at this bifurcation the Lyapunov exponents separate, the largest at 0 , 

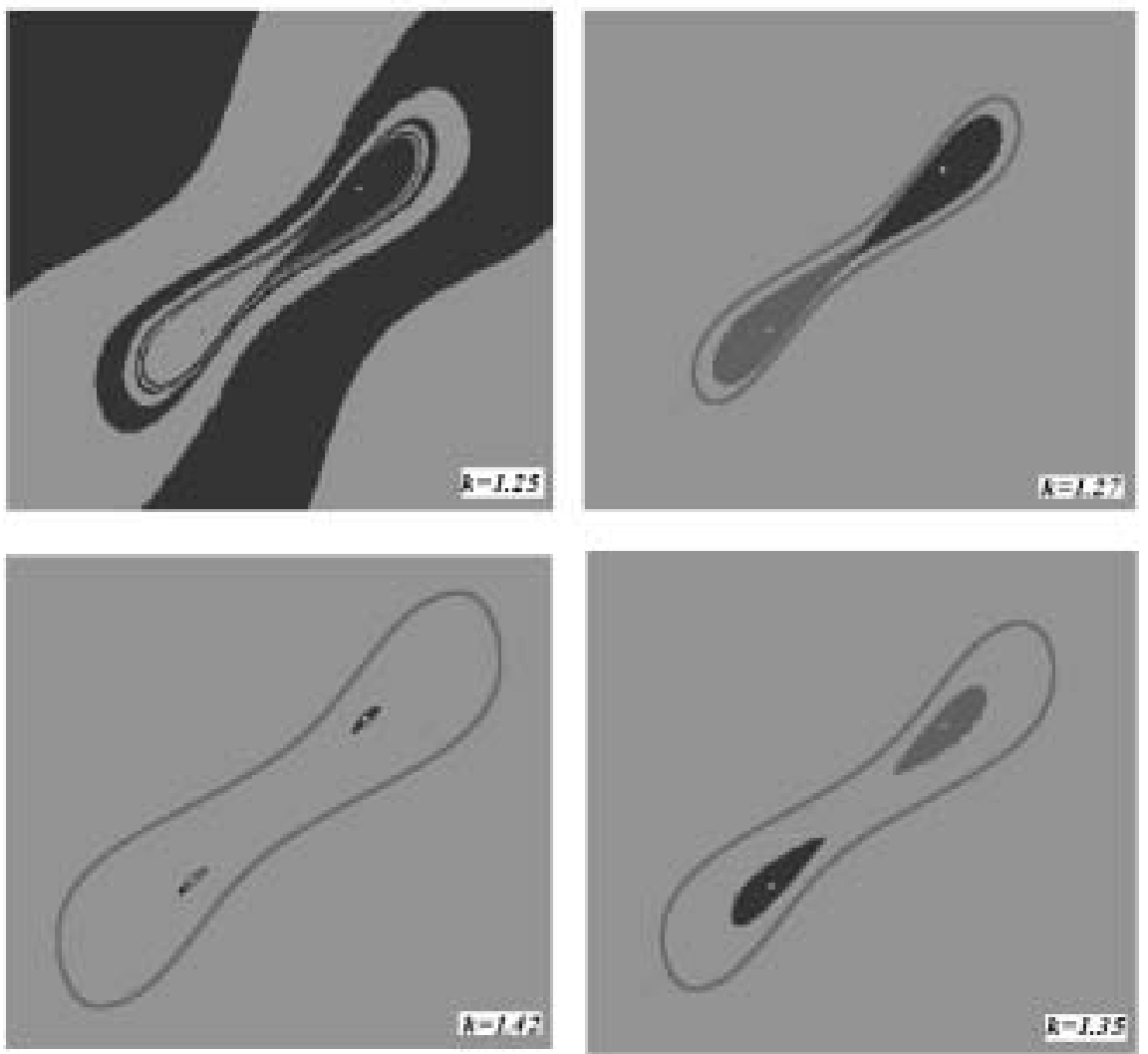

Figure 5: Basins from upper-left, clockwise: $k=1.244,1.27,1.35,1.42$. 
representing motion on the invariant curve, the other negative, representing the attracting property of the curve.

In Figure 6, left $k \in(1.258,1.2595)$, the exponents are calculated over 500 iterations. There seems to be some evidence of chaotic transients, as we would expect for the saddle connection, but these disappear before reaching 5000 iterations (the time range used in Figure 2). The next change occurs
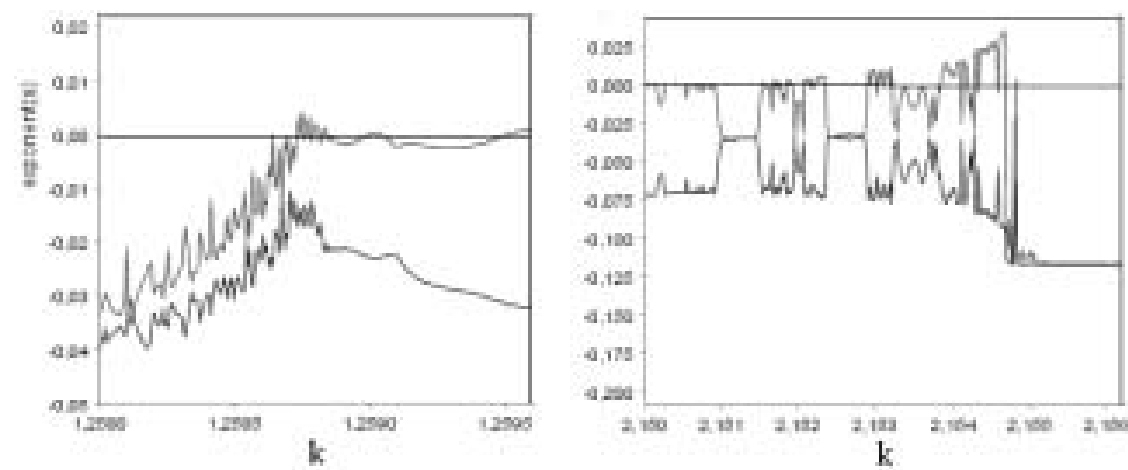

Figure 6: Lyapunov exponents: left, $k \in(1.258,1.2595)$; right, $k \in$ $(2.15,2.156)$.

between upper right and lower right, in which a homoclinic bifurcation of $\mathcal{Y}$ gives rise to a double homoclinic loop and $\Gamma_{u}$ breaks into two repelling curves forming the disjoint basin boundardies $B\left(\bar{Y}_{1}\right), B\left(\bar{Y}_{2}\right)$. In this bifurcation, over a narrow interval of parameter values a homoclinic tangency (in which $w^{u}(\mathcal{Y})$ comes to touch $w^{s}(\mathcal{Y})$ ) is followed by a transversal crossing of the manifolds and a second homoclinic tangency $\left(w^{\mu}(\mathcal{Y})\right.$ is tangent on the opposite side of $w^{s}(\mathcal{Y})$ ). Recall that the stable manifold is the separatrix for the basins of $\bar{Y}_{1}$ and $\bar{Y}_{2}$. The unstable manifold branches of $w^{u}(\mathcal{Y})$ are provided in Figure 7 for the standard parameter constellation and $k=1.289$, above; $k=1.29$, below. Between these values $w^{u}(\mathcal{Y})$ becomes tangent, then crosses, and becomes tangent again to $w^{s}(\mathcal{Y})$. After the homoclinic bifurcation, trajectories with initial conditions close to $\mathcal{Y}$ converge to $\Gamma_{s}$ rather than $\bar{Y}_{1}$ or $\bar{Y}_{2}$. That is, economies starting close to the Samuelsonian equilibrium move away and fluctuate around it.

Finally, the two loops of $\Gamma_{u}$ shrink around $\bar{Y}_{1}$ and $\bar{Y}_{2}$ as $k$ is increased until, at $k=1.429$ (just beyond the value used in Figure 5, lower left), the fixed points lose stability through subcritical Neimark-Sacker bifurcations as 

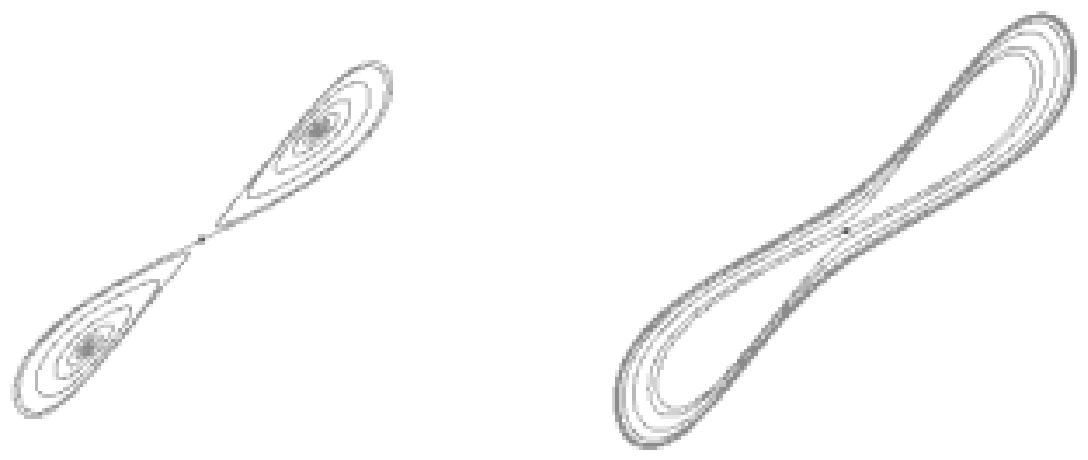

Figure 7: Unstable manifold of $\mathcal{Y}$ : above, $k=1.289$; below, $k=1.29$.

the modulus of the complex, conjugate eigenvalues reaches one. From this value until just before $k=2.13$ all attractors lie on the increasing amplitude invariant curve, $\Gamma_{s}$, to which all initial conditions are attracted.

From invariant curve to period- 8 cycle. The last type of periodic behavior becomes visible at around $k=2.13$. We describe the scenario with reference to Figure 8 , where the basins of attraction are simulated as $k$ increases, starting upper-left and moving clockwise: $k=2.128,2.13,2.15,2.17$. The state space has been enlarged with respect to previous figures to $Y \in(0,10,000)$, as the invariant curve has blown up considerably. The initial conditions for Figure 2 are slightly southwest of center. A saddle-node bifurcation takes place between $k=2.128$ and $k=2.13$. In the upper-left there is still the single attracting invariant curve on which all trajectories eventually lie. After the bifurcation, upper-right, $\Gamma_{s}$ is still attracting for all initial conditions within in it, but most others are attracted to a period- 8 cycle which has appeared around the invariant circle. The basin pieces for the cycle $B(C)$ expand, the basin $B\left(\Gamma_{s}\right)$ shrinks until, by $k=2.17$, the invariant curve has disappeared and all further attractors are periodic. For the propensity to consume out of expected income at $b=0.8$ the last attractor, a period- 8 cycle, becomes unstable around $k=2.53$.

The invariant curve $\Gamma_{s}$ is destroyed and the aperiodic fluctations disappear through the heteroclinic loop sequence described earlier. Starting from coexistence in upper-right, the periodic points and associated saddle points are very near to each other and lie on the boundaries of the basin of attrac- 
tion for the focus cycle $B(C)$. The branches of the stable manifolds of the saddle cycle serve as separatrix between $B(C)$ and $B\left(\Gamma_{s}\right)$. The outer branch of the unstable manifold of the saddle leads to the focus cycle, the inner branch leads to the invariant curve. As $k$ is increased, the inner unstable branch of the saddle point $i$ becomes tangential to the inner stable branch of nearby saddle point $j$, and this happens all around the cycle. This heteroclinic tangency starts a tangle, followed by a transversal crossing of these branches and another heteroclinic tangency. Transversal crossings are usually associated with chaotic repellers and long chaotic transients. A hint of this can be seen in Figure 3 as there is a slight rise in the Lyapunov characteristic exponent near the bifurcation interval, There are clearly chaotic transients evident in Figure 6, right, which are calculated over 5000 iterations and $k \in(2.15,2.156)$. At the end of the tangle the branches are switched in position. The unstable branches of the saddle point $i$ tend to the nearby stable foci (to the right and left, $h$ and $j$ ) forming a heteroclinic saddle-focus connection that leaves no initial condition leading to $\Gamma_{s}$.

For $b=0.8$ this is the end of the story. Had we fixed the propensity to consume at some other level, slightly above or below for example (refer again, to Figure 2, right), the sequence would have continued with another heteroclinic saddle-focus connection forming from the outer branches of the saddle points. This connection would be an invariant closed curve, enveloping and destroying the stable focus cycle. Still higher values of $b$ would have avoided the period- 8 cycle altogether and ended with the first invariant curve becoming unstable.

\subsection{Conclusions}

Samuelson's linear multiplier-accelerator model is a classic example of a business cycle model based on the combined effects of the multiplier and acclerator principles. The equations are simple and the linear dynamics are completely understood. It is interesting to see how these dynamics change under a simple alteration to the consumption hypothesis: expenditures are a function of expected income rather than realized last period income and there are two types of expections (each a linear function of last period income). The aggregate expected income is a nonlinear combination of extrapolative and reverting expectation rules. The equilibrium of Samuelson's model is also a fixed point of the extended model, but other limit sets exist. A comparison of the dynamics of the linear multiplier-accelerator model and the 

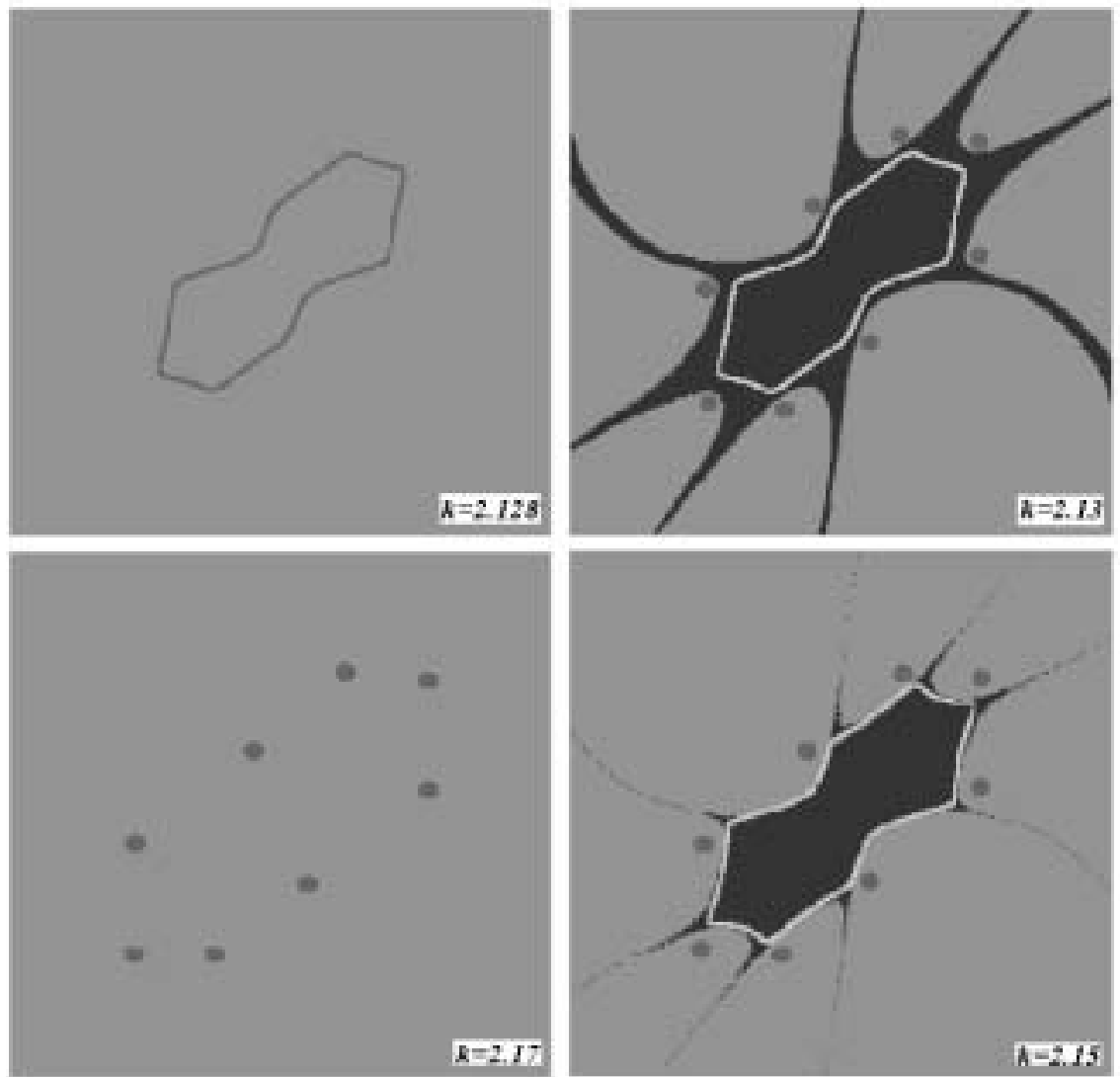

Figure 8: Basins from upper-left, clockwise: $k=2.128,2.13,2.15,2.17$. 
nonlinear expectations-multiplier-accelerator model brings to light essential differences.

1. As regards the equilibrium of Samuelson's model, the stability conditions on $\mathcal{Y}$ are more restrictive in the nonlinear model.

2. However, with nonlinear expectations, local stability of a fixed point may be lost while global stability continues in the form of:

(a) convergence to either of 2, co-existing stable fixed points

(b) a periodic or quasiperiodic sequence of points lying on a closed curve.

3. In fact, over the parameter space $(k, b)$ the nonlinear model has a much larger area characterized by attractors, under reasonable values for the extra parameters and persistent oscillations are a generic possibility in the nonlinear model.

The last characteristic is of special importance given that the phenomenon under study is the business cycle. Moreover this was accomplished by allowing consumption to depend on expectations and expectations to be heterogeneous, that is, by creating a more realistic economic context.

\section{Appendix}

Substituting the expectations formation hypotheses (9) and (10), the expectations weight hypothesis (11) into the aggregate expectations operator (8) 
the complete system (13) is

$$
\begin{aligned}
Y_{t}=I_{a}+ & b(1+k)\left[\left(\frac{1}{1+\gamma^{2}\left(\frac{Y_{t-1}-\mathcal{Y}}{\mathcal{Y}}\right)^{2}}\right)\left(Y_{t-1}+\mu_{1}\left(Y_{t-1}-\mathcal{Y}\right)\right)+\right. \\
& \left.+\left(1-\frac{1}{1+\gamma^{2}\left(\frac{Y_{t-1}-\mathcal{Y}}{\mathcal{Y}}\right)^{2}}\right)\left(Y_{t-1}+\mu_{2}\left(\mathcal{Y}-Y_{t-1}\right)\right)\right] \\
-b k & {\left[\left(\frac{1}{1+\gamma^{2}\left(\frac{Z_{t-1}-\mathcal{Y}}{\mathcal{Y}}\right)^{2}}\right)^{(} Z_{t-1}+\mu_{1}\left(Z_{t-1}-\mathcal{Y}\right)\right)+} \\
& \left.+\left(1-\frac{1}{1+\gamma^{2}\left(\frac{Z_{t-1}-\mathcal{Y}}{\mathcal{Y}}\right)^{2}}\right)\left(Z_{t-1}+\mu_{2}\left(\mathcal{Y}-Z_{t-1}\right)\right)\right] \\
Z_{t}=Y_{t-1} &
\end{aligned}
$$

The Jacobian matrix calculated in either of the fixed points $\bar{Y}_{i} i=1,2$ is

$$
\begin{gathered}
J\left(\bar{Y}_{i}\right)=\left(\begin{array}{cc}
b(1+k) \Phi & -b k \Phi \\
1 & 0
\end{array}\right) \\
\Phi=\frac{-2 \mathcal{Y}^{2} \gamma^{2}\left(\bar{Y}_{i}-\mathcal{Y}\right)^{2}\left(\mu_{1}+\mu_{2}\right)}{\left(\mathcal{Y}^{2}+\gamma^{2}\left(\bar{Y}_{i}-\mathcal{Y}\right)^{2}\right)^{2}}+\frac{\mathcal{Y}^{2}\left(\mu_{1}+\mu_{2}\right)}{\mathcal{Y}^{2}+\gamma^{2}\left(\bar{Y}_{i}-\mathcal{Y}\right)^{2}}+1-\mu_{2}
\end{gathered}
$$

\section{References}

Agliari, A., Bischi, G. and Gardini, L., 200, "Some methods for the global analysis of business cycle models in discrete time"

Agliari, A. and Dieci, R., 2006, "Coexistence of attractors and homoclinic loops in a Kaldor-like business cycle model"

Brock, W. and Hommes. C., 1998, "Heterogeneous beliefs and routes to chaos in a simple asset pricing model", Journal of Economic Dynamics and Control 22: 1235-1274

Day, R. and Huang, W., 1990, "Bulls, bears and market sheep" Journal of Economic Behavior and Organization 14: 299-329 
De Grauwe, P., Dewachter, H. and Embrechts, M., 1993, Exchange Rate Theory - Chaotic Models of Foreign Exchange Markets, Blackwell, Oxford.

Flieth, B. and Foster, J., 2002, "Interactive expectations", Journal of Evolutionary Economics 12: 375-395

Gandolfo, G., 1996, Economic Dynamics, 3rd edn. Springer-Verlag, New York.

Hicks, J.R., 1950, A Contribution to the Theory of the Trade Cycle, Oxford University Press, Oxford

Hommes, C., 1995, "A reconsideration of Hicks' nonlinear trade cycle model", Structural Change and Economic Dynamics 6: 435-459

Hohnisch, M. Pittnauer, S., Solomon, S. and Stauffer D., 2005, "Socioeconomic interaction and swings in business confidence indicators", Physica A 345: 646-656

Ito, T., 1990, "Foreign exchange rate expectations: Micro survey data", American Economic Review 80, 434-449

Kahneman, D., Slovic, P. and Tversky, A, 1986, Judgment under Uncertainty: Heur- istics and Biases, Cambridge University Press, Cambridge

Kirman, A., 1993, "Ants, rationality, and recruitment", Quarterly Journal of Economics 108: 137-156

Lux, T. and Marchesi, M., 2000, "Volatility clustering in financial markets: A micro-simulation of interacting agents", International Journal of Theoretical and Applied Finance 3: 675-702

Puu, T., Gardini, L. and Sushko, I., 2005, "A Hicksian multiplier-accelerator model with floor determined by capital stock", Journal of Economic Behavior and Organization 56: 331-348

Samuelson, P. ,1939, "Interactions between the multiplier analysis and the principle of acceleration", Review of Economic Statistics 21: 75-78

Simon, H., 1955, "A behavioral model of rational choice", Quarterly Journal of Economics 9: 99-118 
9 Expectations and the Multiplier-Accelerator Model

Shiller, R., 2000, Irrational Exuberance, Princeton University Press, Princeton

Smith, V., 1991, Papers in Experimental Economics, Cambridge University Press, Cambridge

Sonnemans, J., Hommes, C., Tuinstra, J. and van de Velden, H., 2004, "The instability of a heterogeneous cobweb economy: a strategy experiment on expectation formation", Journal of Economic Behavior and Organization 54: 453-481

Takagi, S., 1991, "Exchange rate expectations: A survey of survey studies", IMF Staff Papers 38: 156-183

Westerhoff, F. ,2005, "Samuelson's multiplier-accelerator model revisited", Applied Economics Letters (in press). 